\title{
Confiabilidade intra e interexaminadores da aplicação da Escala Motora Infantil de Alberta (EMIA) em ambulatório de seguimento de recém-nascidos de risco
}

\author{
Intra- and inter-examiner reliability of Alberta Infant Motor Scale application in follow-up \\ ambulatory of at-risk newborns
}

Fiabilidad intraevaluadores e interevaluadores de la aplicación de la Escala Motora Infantil de Alberta (EMLA) en un seguimiento ambulatorio de recién nacidos de riesgo

Olivia Ramalho', Luciana Sayuri Sanada², Natália Alves Menegol ${ }^{3}$, Sheila Cristina da Silva Pacheco ${ }^{4}$, Anelise Sonza ${ }^{5}$, Dayane Montemezzo ${ }^{6}$

\begin{abstract}
RESUMO I A prematuridade é fator de risco para atraso do desenvolvimento motor, e recomenda-se o acompanhamento desses lactentes nos primeiros dois anos de vida. Verificar a confiabilidade intra e interexaminadores da Escala Motora Infantil de Alberta (EMIA) em ambulatório de seguimento de recém-nascidos de risco de uma maternidade pública. Estudo prospectivo realizado em ambulatório de seguimento de recém-nascidos de risco. As avaliações do desenvolvimento motor foram realizadas por meio da EMIA, por dois avaliadores previamente treinados. O Coeficiente de Correlação Intraclasse (CCl) foi utilizado para análise das confiabilidades. Para a comparação entre as avaliações intraexaminadores foi realizado o Teste $T$ pareado ou Teste de Wilcoxon. O Teste $T$ independente foi utilizado para comparar as avaliações interexaminadores. A correlação entre as variáveis foi analisada a partir do Teste de Pearson ou Spearman. Para avaliar a concordância entre os escores foi realizada análise de Bland Altman. Foram avaliados 31 recém-nascidos pré-termo (RNPT) com idade corrigida média de 8,47 $\pm 4,49$. Não houve diferença
\end{abstract}

significativa entre as avaliações intraexaminadores e interexaminadores. Os valores de $\mathrm{CCl}$ se mantiveram acima de 0,88 para a confiabilidade intraexaminadores e interexaminadores. Os escores apresentaram alta concordância, analisada por meio do teste de Bland Altman. EMIA apresentou adequada confiabilidade intra e interexaminadores para avaliação e acompanhamento de RNPT até 18 meses em ambulatório de seguimento de lactentes de risco.

Descritores | Desenvolvimento Motor; EMIA; Confiabilidade.

\begin{abstract}
Prematurity is a risk factor for delayed motor development, and it is recommended to monitor these infants in the first two years of life. To verify the properties of intra and inter-examiner measurements of AIMS in an outpatient follow-up clinic for newborns at risk in a public maternity hospital. Prospective study conducted in an outpatient follow-up of high-risk newborns. The Intraclass Correlation Coefficient (ICC) was used to analyze reliability. To compare the intra-examiner evaluations, the paired T-test or Wilcoxon
\end{abstract}

Universidade do Estado de Santa Catarina (Udesc) - Florianópolis (SC), Brasil. E-mail.: olicara@hotmail.com. Orcid: 0000-0001-8796-1231 Universidade do Estado de Santa Catarina (Udesc) - Florianópolis (SC), Brasil. E-mail: luciana.sanada@udesc.br. Orcid: 0000-0002-6530-6831

3Universidade do Estado de Santa Catarina (Udesc) - Florianópolis (SC), Brasil. E-mail: nataliamenegol@hotmail.com. Orcid: 0000-0003-3407-9776

${ }^{4}$ Universidade do Estado de Santa Catarina (Udesc) - Florianópolis (SC), Brasil. E-mail: scspacheco@gmail.com. Orcid: 0000-0002-3604-9987

5Universidade do Estado de Santa Catarina (Udesc) - Florianópolis (SC), Brasil. E-mail: anelise.sonza@udesc.br. Orcid: 0000-0003-0056-4984

${ }^{6}$ Universidade do Estado de Santa Catarina (Udesc) - Florianópolis (SC), Brasil. E-mail: dayane.montemezzo@udesc.br. Orcid: 0000-0001-7680-8223 
test was performed. The independent T-test was used to compare inter-examiner assessments. The correlation between variables was analyzed using the Pearson or Spearman test. The Bland Altman test was performed to assess the concordance between the scores. 31 preterm infants with 8,47 \pm 4,49 of corrected age were evaluated. There was no significant difference between the evaluations intra and inter-examiner. The ICC values remained above 0.88 for both intra and inter-examiner evaluation. The scores showed high agreement. AIMS has intra- and inter-examiner reliability for assessing and monitoring preterm newborns for up to 18 months in a follow-up clinic.

Keywords | Motor Development; AIMS; Reliability.

RESUMEN | La prematuridad es un factor de riesgo de retraso en el desarrollo motor de los lactantes y se recomienda la monitorización de ellos durante los dos primeros años de vida. Verificar la fiabilidad intraevaluadores e interevaluadores de la Escala Motora Infantil de Alberta (EMIA) en un seguimiento ambulatorio de recién nacidos de riesgo en una maternidad pública brasileña. Estudio prospectivo realizado en un seguimiento ambulatorio de recién nacidos de riesgo. Para evaluar el desarrollo motor, la EMIA fue empleada por dos evaluadores previamente capacitados. Se utilizó el coeficiente de correlación intraclase $(\mathrm{CCl})$ para analizar la fiabilidad. Para comparar las evaluaciones intraevaluadores, se utilizó la prueba T pareada o la prueba de Wilcoxon. La prueba T independiente se utilizó para comparar las evaluaciones interevaluadores. La correlación entre las variables se analizó mediante la prueba de Pearson - Spearman. Para evaluar la concordancia entre los puntajes, se aplicó el análisis de Bland Altman. Se evaluaron a 31 recién nacidos pretérmino (RNPT) con un promedio de edad media corregida de $8,47 \pm 4,49$. No hubo diferencias significativas entre las evaluaciones intraevaluadores e interevaluadores. Los valores de $\mathrm{CCl}$ se mantuvieron por encima de 0,88 para la fiabilidad intraevaluadores e interevaluadores. Los puntajes mostraron un alto nivel de concordancia, que se analizó mediante el Bland Altman. La EMIA apuntó una adecuada fiabilidad intra e interevaluadores para evaluar y monitorear los RNPT hasta 18 meses en seguimiento ambulatorio de lactantes de riesgo. Palabras clave / Desarrollo Motor; EMIA; Fiabilidad.

\section{INTRODUÇÃO}

A prematuridade é uma das maiores causas de mortalidade e morbidade infantil, tanto no período neonatal, como na primeira infância, devido a imaturidade geral que pode levar a alterações e disfunções sistêmicas, além de atrasos cognitivo, motor, comunicativo, comportamental, de aprendizado e sensorial ${ }^{1,2}$. O Brasil encontra-se em $10^{\circ}$ lugar no ranking mundial de nascimentos prematuros, apresentando um índice de $11,5 \%$ de nascimentos prematuros, o que gera aumento do custo diário de internação em unidade de terapia intensiva (UTI) neonatal, de acordo com estudos realizados ${ }^{1,3}$.

$\mathrm{Na}$ UTI neonatal, além dos fatores de risco biológicos, devido à baixa idade gestacional que implicam em diversas alterações dos sistemas essenciais ${ }^{4}$, sabe-se que o recém-nascido pré-termo (RNPT) está exposto a fatores ambientais que também irão contribuir para alterações no seu desenvolvimento motor. Internado na UTI neonatal, o RNPT é submetido a uma série de procedimentos invasivos e dolorosos, espaço físico restrito, ausência de estímulos adequados, rotina hospitalar que afetam a sua mobilidade, contribuem para um desenvolvimento motor atrasado e deficiente ${ }^{4}$.

Detectar esses fatores e minimizá-los de forma precoce, assim como realizar uma avaliação criteriosa nos primeiros anos de vida, e identificar distúrbios no desenvolvimento motor, permite determinar uma intervenção adequada que possibilite que crianças com atrasos possam seguir o mesmo ritmo de aquisições de uma criança com desenvolvimento motor normal ${ }^{4,5}$. Evidências também demonstram que, durante os primeiros dois anos de vida, um programa de intervenção precoce se mostra mais efetivo, devido à alta neuroplasticidade do RNPT e, por isso, após o período de internação, recomenda-se o acompanhamento multiprofissional em ambulatório de seguimento de risco até os dois primeiros anos de vida ${ }^{6,7}$.

As escalas para avaliação do desenvolvimento motor de lactentes como a Bayley Scales of Infant Development e a Peabody Developmental Motor Scales, apesar de serem validadas, confiáveis e testadas em diversos países, têm alto custo e necessitam de treinamento específico ${ }^{8}$. A Escala Motora Infantil de Alberta (EMIA), entretanto, tem sido muito utilizada na avaliação das habilidades motoras por ser de fácil e rápida aplicação, e ter se 
mostrado sensível para a detecção de déficits motores ${ }^{9}$. Foi desenvolvida por Piper e Darrah, em 1994, como uma escala de medida observacional da função motora ampla utilizada em lactentes a termo e pré-termo acima de quarenta semanas gestacionais, até a aquisição da marcha independente ${ }^{10}$. A EMIA foi validada para a população brasileira por Valentini e Saccani ${ }^{11}$, e os resultados demostraram adequada confiabilidade e validade para emprego no acompanhamento do desenvolvimento de lactentes brasileiros.

Estudos anteriores analisaram algumas propriedades de medida da EMIA, porém as propriedades de medida em lactentes prematuros acompanhados em ambulatório de seguimento de risco não foram estudadas. Sendo assim, o objetivo deste estudo foi verificar a confiabilidade intra e interexaminadores da Escala Motora Infantil de Alberta em um ambulatório de seguimento de recém-nascidos de risco.

\section{METODOLOGIA}

Estudo prospectivo realizado no ambulatório de seguimento de recém-nascidos de risco de uma maternidade de referência do sul do Brasil. Os responsáveis foram informados sobre o objetivo do estudo e dos procedimentos a serem realizados com os lactentes; após estarem de acordo, todos anuíram ao Termo de Consentimento Livre e Esclarecido e ao Termo de Consentimento de Imagem e Vídeo.

Participaram do estudo lactentes de ambos os sexos, entre 0 e 18 meses de idade corrigida, em acompanhamento no ambulatório de seguimento da maternidade; foram excluídos lactentes com história clínica de malformação congênita, síndromes genéticas, hemorragia peri-intraventricular graus III e IV, lactentes instáveis clinicamente, e aqueles que não concluíram o protocolo do estudo. A amostra foi não probabilística e intencional.

A caracterização da amostra foi feita por meio de instrumento próprio para o estudo e respondido pelos responsáveis. As avaliações do desenvolvimento motor foram feitas utilizando o roteiro da EMIA, em ambiente reservado, por dois avaliadores previamente treinados. $\mathrm{O}$ treinamento dos examinadores consistiu em 18 horas de aulas teórico-práticas sobre a aplicação da EMIA, além da experiência prática na aplicação das avaliações com a EMIA em lactentes. A escala contém 58 itens, divididos em quatro subescalas: prono (21 itens), supino (nove itens), sentado (12 itens) e em pé (16 itens). A avaliação é observacional, segue um roteiro próprio analisando três quesitos, saber: o movimento antigravitacional, a descarga de peso e o controle postura $1^{10}$. O escore de um ponto é concedido a cada um dos itens que o lactente realiza e aos anteriores a abertura da janela motora de cada subescala, sendo assim, a somatória das quatro subescalas gera o escore total ${ }^{10}$. Assim, por meio do escore total e da idade corrigida do lactente, é calculado o percentil, sendo lactentes com percentil abaixo de $25 \%$, considerados de risco ${ }^{11,12}$. Em caso de agitação e choro excessivo, ou quaisquer mudanças comportamentais, a avaliação era interrompida até que o lactente voltasse à condição inicial, para não interferir nos resultados.

Foi utilizada uma câmera Nikon Coolpix L120 ${ }^{\circledR}$, posicionada em um tripé ajustado a uma altura de um metro, ângulo de $45^{\circ}$ a 1,5 metros de distância do avaliador e do lactente.

Para o protocolo da confiabilidade interexaminadores, dois avaliadores, analisaram simultaneamente os lactentes marcando sua própria pontuação, porém um examinador realizava contato físico com a criança durante as posturas. O tempo das avaliações foi cronometrado e a ordem dos avaliadores que aplicariam a EMIA foi aleatorizada. Para atender o protocolo da confiabilidade intraexaminadores, as filmagens foram avaliadas novamente pelo mesmo avaliador, entre sete a quinze dias após a primeira avaliação, para reduzir a interferência da memória ${ }^{12}$.

Após as avaliações, os resultados foram tabulados e os dados analisados no Statistical Package for the Social Sciences versão 20.0. Os dados foram apresentados como medidas de tendência central e dispersão, de acordo com a distribuição normal dos dados. Para análise das confiabilidades intra e interexaminadores foi utilizado o Coeficiente de Correlação Intraclasse (CCI). Para a comparação entre as avaliações da confiabilidade intraexaminadores foi utilizado o Teste $\mathrm{T}$ pareado ou de Wilcoxon, conforme a distribuição normal dos dados. $\mathrm{O}$ teste $\mathrm{T}$ independente foi utilizado para a comparação entre as avaliações da confiabilidade interexaminadores. A correlação entre as variáveis foi analisada a partir do teste de Correlação de Pearson ou de Spearman, conforme distribuição normal dos dados. A análise de Bland Altman foi aplicada para analisar se há concordância entre os escores das 
avaliações das confiabilidades intra e interexaminadores a partir da média e desvio-padrão das diferenças e os limites inferior e superior de concordância. $\mathrm{O}$ nível de significância determinado foi $\mathrm{p}<0,05$ para todas as análises estatísticas.

\section{RESULTADOS}

Foram avaliados 31 lactentes com idade corrigida média de 8,47 $\pm 4,49$ meses, sendo 10 avaliações utilizadas para a análise de confiabilidade intraexaminador $1 ; 10$ para a análise de confiabilidade intraexaminador 2; e 11 para a confiabilidade interexaminadores, cujos dados da caracterização dos lactentes encontram-se na Tabela 1.

Os resultados relativos aos escores e percentis da aplicação da EMIA para avaliação das confiabilidades intraexaminadores e interexaminadores estão apresentados na Tabela 2

Os resultados relacionados à confiabilidade intra e interexaminadores estão representados na Tabela 3.

A correlação dos dados, a partir da análise de correlação de Pearson e Spearman, está representada na Figura 1.

A análise qualitativa de concordância dos dados dos escores da EMIA foi representada a partir da análise de Bland Altman, conforme mostra a Figura 2.

Tabela 1. Caracterização dos lactentes participantes da confiabilidade intra e interexaminadores

\begin{tabular}{|c|c|c|c|c|c|}
\hline Variáveis & Intraexaminador $1(n=10)$ & $\begin{array}{c}\text { Intraexaminador } 2 \\
(n=10)\end{array}$ & $\begin{array}{c}\text { Inter examinador } \\
(\mathrm{n}=11)\end{array}$ & $\begin{array}{l}\text { Total } \\
(n=31)\end{array}$ & p-valor \\
\hline Sexo & $6 \mathrm{M} / 4 \mathrm{~F}$ & $8 \mathrm{M} / 2 \mathrm{~F}$ & $6 \mathrm{M} / 5 \mathrm{~F}$ & $20 \mathrm{M} / 11 \mathrm{~F}$ & - \\
\hline IG (semanas) & $33,16(3,50)$ & $33,21(2,32)$ & $32,80(1,62)$ & $33,06(2,69)$ & 0,89 \\
\hline $\mathrm{ICr}$ (meses) & $10,08(4,87)$ & $7,13(4,48)$ & $9,83(4,17)$ & $8,95(3,89)$ & 0,31 \\
\hline ICo (meses) & $8,37(4,71)$ & $5,52(4,33)$ & $8,03(4,23)$ & $8,47(4,29)$ & 0,06 \\
\hline PN (g) & $2146,25(865,46)$ & $1935(831,75)$ & $1790(445,60)$ & $1900[1013]$ & 0,03 \\
\hline
\end{tabular}

M: masculino; F: feminino; IG: idade gestacional; ICr: idade cronológica; ICo: idade corrigida; PN: peso ao nascer. Dados apresentados pela média e desvio-padrão (DP).

Tabela 2. Confiabilidade intraexaminador nas avaliações 1 e 2 e interexaminador (avaliador 1 e 2) da escala EMIA aplicada em lactentes

\begin{tabular}{|c|c|c|c|c|c|c|c|}
\hline & & \multirow{2}{*}{ EMIA } & \multirow{2}{*}{ Avaliação 1} & \multirow{2}{*}{ Avaliação 2} & \multicolumn{2}{|c|}{ IC $95 \%$} & \multirow{2}{*}{ p } \\
\hline & & & & & LI & LS & \\
\hline \multirow{4}{*}{$\begin{array}{l}\text { Confiabilidade } \\
\text { intraexaminador }\end{array}$} & \multirow[t]{2}{*}{ Avaliador 1} & Escore & $23,60 \pm 17,6$ & $23,10 \pm 17$ & & & $0,55^{+}$ \\
\hline & & Percentil & $9,50 \pm 2,7$ & $9,30 \pm 2,7$ & $-0,46$ & 0,86 & $0,51^{*}$ \\
\hline & \multirow[t]{2}{*}{ Avaliador 2} & Escore & $32,9 \pm 20$ & $32 \pm 20,7$ & & & $0,12^{+}$ \\
\hline & & Percentil & $2,40 \pm 1$ & $2,4 \pm 1,1$ & & & $1,00^{+}$ \\
\hline \multirow{3}{*}{\multicolumn{2}{|c|}{$\begin{array}{l}\text { Confiabilidade interexaminador } \\
\text { Escore } \\
\text { Percentil }\end{array}$}} & & Avaliador 1 & Avaliador 2 & & & \\
\hline & & Escore & $34,64 \pm 17$ & $33,73 \pm 16,9$ & $-14,16$ & 15,98 & $0,90^{\#}$ \\
\hline & & Percentil & $6,36 \pm 2,6$ & $6,09 \pm 2,9$ & $-2,17$ & 2,72 & $0,82^{\#}$ \\
\hline
\end{tabular}

EMIA: Escala motora infantil de Alberta; LI: Limite inferior; LS: Limite superior; IC: Intervalo de confiança da diferença; dados apresentados por média \pm desvio-padrão; $p=p$ valor; ${ }^{+}$realizado teste de Wilcoxon; 'realizado Teste t pareado; "realizado Teste T.

Tabela 3. Análise da confiabilidade da AIMS

\begin{tabular}{|c|c|c|c|c|}
\hline & & $\mathrm{CCl}$ & $\begin{array}{c}95 \% \mathrm{CCl} \\
\text { LI-LS }\end{array}$ & $\mathbf{P}$ \\
\hline \multirow{3}{*}{ Escore } & 1 & 1,00 & $1,00-1,00$ & $<0,001$ \\
\hline & 2 & 0,99 & $0,97-1,00$ & $<0,001$ \\
\hline & $1-2$ & 0,99 & $0,97-1,00$ & $<0,001$ \\
\hline \multirow{3}{*}{ Percentil } & 1 & 0,89 & $0,63-0,97$ & $<0,001$ \\
\hline & 2 & 0,88 & $0,59-0,97$ & $<0,001$ \\
\hline & $1-2$ & 0,96 & $0,86-0,99$ & $<0,001$ \\
\hline
\end{tabular}

1: avaliador 1; 2: avaliador 2; 1-2: interexaminadores; CCl: Coeficiente de Correlação Interclasse; LI: Limite inferior; LS: Limite superior; p: p-valor 
Intraexaminador 1
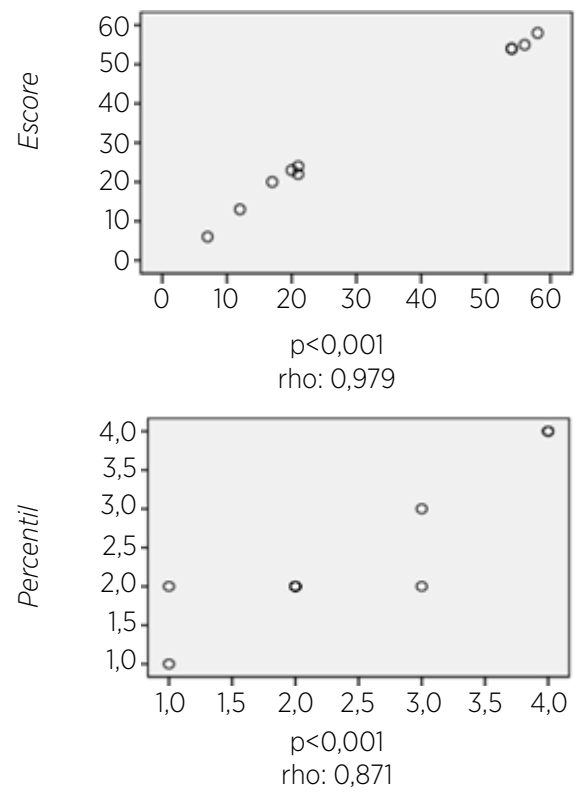

Intraexaminador 2
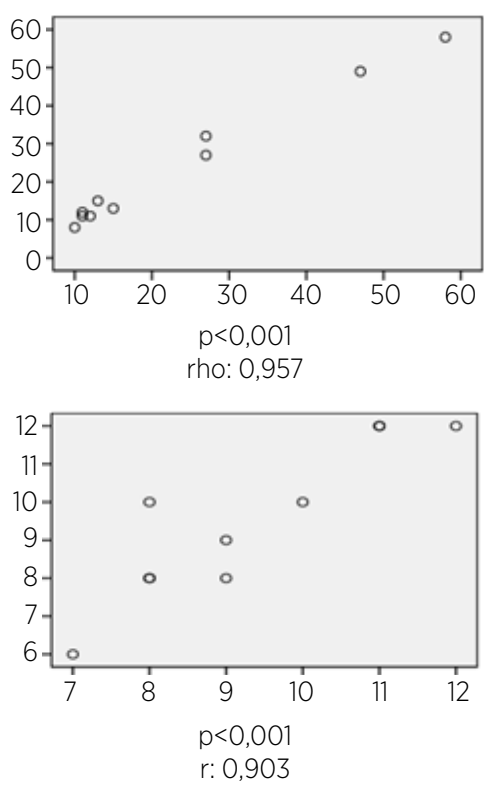

Interexaminadores
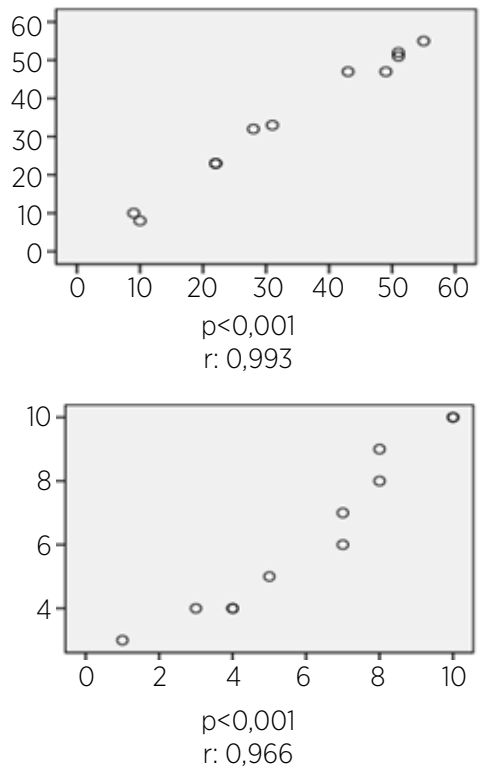

Figura 1. Gráficos das análises de correlação entre os escores 1 e 2; e os percentis 1 e 2

Dados da primeira e segunda avaliação representados no eixo x e y, respectivamente. p: p-valor; r: coeficiente de Pearson; rho: coeficiente de Spearman.
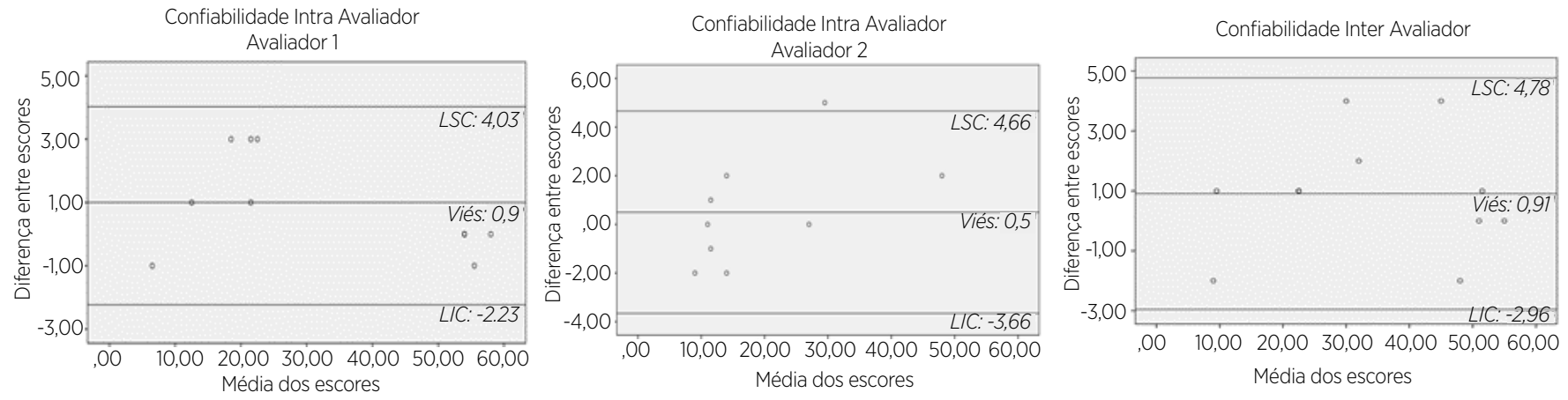

Figura 2. Análise qualitativa de concordância dos dados dos escores representada a partir do teste de Bland Altman

\section{DISCUSSÃO}

Este estudo trouxe como diferencial as análises de concordância, homogeneidade, correlação e confiabilidade entre os dados da EMIA obtidos por dois examinadores. Assim, estes resultados mostraram que a EMIA é adequada para avaliar o desenvolvimento motor e identificar possíveis atrasos motores de lactentes acompanhados em um ambulatório de seguimento de risco de uma maternidade de referência do sul do Brasil. Mostrou-se também, que o treinamento simples foi apropriado para preparar os profissionais para avaliação desses lactentes. Grande parte dos prematuros, apesar de serem considerados de risco, após a alta hospitalar acabam não sendo acompanhados com a frequência recomendada de, ao menos duas vezes, entre 3-5 meses de idade corrigida, aos 12 e 24 meses de idade corrigida ${ }^{13}$. Nesse contexto, quando há o atraso no desenvolvimento motor, este acaba não sendo identificado, podendo acarretar, a longo prazo, em problemas de coordenação motora, déficit de atenção, bem como atrasos em performance cognitiva e escolar ${ }^{14,15}$. Portanto, nos primeiros anos de vida, os lactentes prematuros precisam de avaliações periódicas com escalas padronizadas, para que qualquer mudança no desenvolvimento global da criança seja detectada de forma precoce ${ }^{7}$.

A padronização das escalas ocorre por meio da avaliação das propriedades de medida, sendo a confiabilidade uma delas, visto que aborda aspectos sobre coerência, precisão, estabilidade, equivalência e homogeneidade de um instrumento, e principalmente se tratando de uma 
população de risco ${ }^{16}$. No presente estudo os valores de percentil demonstraram que não houve diferença significativa entre as avaliações $\left(\mathrm{LI}_{\mathrm{p}}=0,34 \%\right.$ e $\left.\mathrm{LS}_{\mathrm{p}}=0,34 \%\right)$. Em relação à confiabilidade interexaminadores, os valores de IC 95\% mostram que não há diferença significativa entre as avaliações tanto em relação ao escore $\left(\mathrm{LI}_{\mathrm{e}}=-0,42\right.$ pontos e $\mathrm{LS}_{\mathrm{e}}=2,23$ pontos) como ao percentil $\left(\mathrm{LI}_{\mathrm{p}}=-0,25 \%\right.$ e $\left.\mathrm{LS}_{\mathrm{p}}=-0,80 \%\right)$. Mais ainda, no presente estudo, o CCI do percentil da EMIA, tanto intra como interexamiandor se mantiveram acima de 0,88 , enquanto para o escore da escala o CCI foi $\geq 0,99$. O CCI, que é uma medida de avaliação de concordância entre os dados, apresentou em nosso estudo valores acima de 0,75 , e aproximando-se de 1 , representando alta confiabilidade para aplicação de testes de acordo com a literatura ${ }^{12}$. De forma semelhante, Almeida et al. ${ }^{17}$, com o objetivo de avaliar a validade concorrente e a confiabilidade interexaminador da EMIA em prematuros do Rio de Janeiro, verificaram a confiabilidade interexaminadores $\mathrm{da}$ EMIA, com valores satisfatórios de ICC em todas as idades avaliadas, variando de 0,76 a 0,99 . Estes autores verificaram ainda que a validade concorrente entre os escores brutos da EMIA e da Escala Bayley de Desenvolvimento Infantil II tinham uma excelente correlação ( $r=0,97 ; p<0,001$,). Em 2013, Silva et al. ${ }^{18}$ analisaram a confiabilidade da EMIA, em 50 lactentes cearenses nascidas pré-termo e a termo, de faixa etária em torno de 4 meses, cujos resultados mostraram CCI acima de 0,8 (19). Além desses, no estudo de validação da escala para a população gaúcha, a confiabilidade intraavaliador demonstrou uma forte concordância (CCI=0,92-0,99. Entretanto, apesar de vários estudos avaliarem a confiabilidade da EMIA, vale ressaltar que o diferencial desse estudo é a avaliação dessas medidas em lactentes acompanhados em um ambulatório de seguimento de risco, além da análise complementar e Bland Altman.

Estudos recomendam que, além da confiabilidade analisada pelo CCI, a concordância seja avaliada de forma complementar, pois os dados podem indicar uma medida correlacionada mas não concordante ${ }^{18-20}$. Para isso, além do CCI, foi avaliada a concordância entre os examinadores, por meio da análise de Bland Altman. Giavarina ${ }^{20}$ descreve o método de Bland Altman como um método para quantificar a concordância entre duas medidas quantitativas por meio da construção de limites superiores e inferiores, ou seja, por meio de uma análise específica é possível quantificar $o$ viés e uma faixa de concordância entre as medidas ${ }^{21}$. No presente estudo, por meio deste teste estatístico, pode-se observar que a média das diferenças para a análise do intraexaminador $1(\mathrm{p}=0,108)$, intraexaminador $2(\mathrm{p}=0,475)$ e interexaminadores $(\mathrm{p}=0,157)$ se mantiveram próxima de zero, sem diferenças estatisticamente significativas, indicando alta concordância intraexaminador e interexaminador. A amplitude dos limites, tanto inferior como superior, apesar de baixa no presente estudo, deve ser avaliada clinicamente, observando cada lactente avaliado, podendo ser observado, dessa forma, se essa variação será significativa na área clínica ${ }^{19,20}$. Entretanto, observando os limites de concordância, dentre as 31 avaliações, apenas 1 avaliação, da confiabilidade intraexaminador 2 , se apresentou como outlier, se mantendo fora dos limites de concordância ( $\mathrm{LI}=-3,66$ pontos; $\mathrm{LS}=4,66$ pontos), não influenciando os resultados, visto que se recomenda que cerca de $95 \%$ dos pontos se mantenham dentro do recomendado ${ }^{22}$.

Portanto, a partir destes dados, pode-se verificar que para a prática clínica, a avaliação com a EMIA em um ambulatório de lactentes de risco apresenta alta confiabilidade intra e interexaminador, além de ser altamente concordante. Este achado pode ser considerado uma importante contribuição para os fisioterapeutas que atuam em ambulatórios como este no qual o presente estudo foi desenvolvido. Caracteriza-se como um diferencial deste estudo em direção a aplicabilidade clínica da EMIA por diferentes profissionais fisioterapeutas que atuam em ambulatório de seguimentos de $\mathrm{RN}$ de risco, desde que sejam criteriosamente treinados para desempenhar esta atividade.

No entanto, uma limitação do estudo foi a dificuldade na seleção da amostra. Grande parte dos pais e/ou responsáveis que eram contatados não quiseram comparecer à avaliação fisioterapêutica, por mais que esta fosse recomendada pelos médicos pediatras do ambulatório de seguimento, e por esse motivo, o tamanho amostral do estudo não foi satisfatório. A distribuição homogênea da faixa etária da amostra também é uma possível limitação, recomendando-se assim que estudos futuros realizem a análise de concordância de Bland Altman em uma maior amostra e em novas faixas etárias. Sugere-se ainda para futuros estudos, análises semelhantes à do presente estudo considerando os subescores das posições prona, supina, sentada e em pé,já que o escore total de quando analisadas em conjunto pode ser mascarado mesmo que haja diferença entre as subescalas.

\section{CONCLUSÃO}

Este estudo mostrou que a EMIA apresenta alta confiabilidade intra e interexaminadores com prematuros de risco de até 18 meses, e sugere-se que pode ser 
aplicada como forma de avaliação e acompanhamento do desenvolvimento motor de recém-nascidos pré-termo em acompanhamento em ambulatórios de seguimento de risco por profissionais treinados.

\section{REFERÊNCIAS}

1. Leal C, Esteves-Pereira AP, Nakamura-pereira M, Torres JA, Theme-filha M, Maria R, et al. Prevalence and risk factors related to preterm birth in Brazil. Reprod Health. 2016;13(Suppl 3):164-74. doi: 10.1186/s12978-016-0230-0

2. Voller SMB. Follow-Up care for high-risk preterm. Pediatr Ann. 2018;47(4):142-6. doi: 10.3928/19382359-20180325-03

3. Viana Filho JR, Pássari IA, Niveiros Filho SI. Gestão de custos hospitalares : um estudo de caso no Hospital Santa Casa de Misericórdia e Maternidade de Rondonópolis - MT. In: Machado MCK, editor. Controladoria, Gestão de Custos e Finanças. Ponta Grossa: Atena; 2017. p. 150-170. doi: 10.22533/at.ed.22318271210

4. Panceri C, Ruttnig K, Pereira G, Valentini NC, Helena R, Salazar A. A influência da hospitalização no desenvolvimento motor de bebês internados no Hospital de Clínicas de Porto Alegre. Clin Biomed Res [Internet]. 2012 [cited 2020 May 10];32(2):161-8. Available from: https://seer.ufrgs.br/hcpa/article/view/25819

5. Guimarães CLN, Reinaux CM, Botelho ACG, Lima GMS, Cabral Filho JE. Desenvolvimento motor avaliado pelo Test of Infant Motor Performance: comparação entre lactentes pré-termo e a termo. Rev Bras Fisioter. 2011;15(5):357-62. doi: 10.1590/ S1413-35552011005000021

6. Khurana S, Kane AE, Brown SE, Tarver T, Dusing SC. Effect of neonatal therapy on the motor, cognitive, and behavioral development of infants born preterm: a systematic review. Dev Med Child Neurol. 2020;62(6):684-92. doi: 10.1111/dmcn.14485

7. Sociedade Brasileira de Pediatria. Seguimento Ambulatorial do Prematuro de Risco [Internet]. Porto Alegre; 2010 [cited 2020 May 10]. Available from: https://www.sbp.com.br/fileadmin/ user_upload/pdfs/Seguimento_prematuro_ok.pdf

8. Albuquerque PL, Lemos A, Guerra MQDF, Eickmann SH. Accuracy of the Alberta Infant Motor Scale (AIMS) to detect developmental delay of gross motor skills in preterm infants: A systematic review. Dev Neurorehabil. 2015;18(1):15-21. doi: 10.3109/17518423.2014.955213
9. Lackovic M, Nikolic D, Filimonovic D, Petronic I, Mihajlovic S, Golubovic Z, et al. Reliability, Consistency and Temporal Stability of Alberta Infant Motor Scale in Serbian Infants. Children. 2020;7(3):16. doi: 10.3390/children7030016

10. Piper MC, Darrah J. Motor Assessment of the Developing Infant. Philadelphia: Saunders; 1994.

11. Valentini NC, Saccani R. Brazilian Validation of the Alberta Infant Motor Scale. Phys Ther. 2012;92(3):440-7. doi: 10.2522/ ptj.20110036

12. Valentini NC, Saccani R. Escala Motora Infantil de Alberta: Validação para uma população gaúcha. Rev Paul Pediatr. 2011;29(2):231-8. doi: 10.1590/S0103-05822011000200015

13. Portney LG. Measurement Revisited: Reliability and Validity Statistics. In: Portney L. Foundations of Clinical Research: Applications to Evidence-Based Practice [Internet]. 4th ed. Philadeplhia: Saunders; 2020 [cited 2020 May 10]. Available from: http://fadavispt.mhmedical.com/content. aspx?aid=1172486561

14. McKinnon K, Huertas-Ceballos A. Developmental follow-up of children and young people born preterm, NICE guideline 2017. Arch Dis Child Educ Pract Ed. 2019;104(4):224. doi: 10.1136/ archdischild-2017-314044

15. Moreira RS, Magalhães LC, Alves CRL. Effect of preterm birth on motor development, behavior, and school performance of schoola-age children: A systematic review. J Pediatr. 2014;90(2):119-34. doi: 10.1016/j.jpedp.2013.05.009

16. Melo WA, Uchimura TT. Perfil e processo da assistência prestada ao recém-nascido de risco no Sul do Brasil. Rev Bras Epidemiol. 2011;14(2):323-37. doi: 10.1590/S1415-790X2011000200013

17. Almeida KM, Dutra MVP, Mello RR, Reis ABR, Martins PS. Validade concorrente e confiabilidade da Alberta Infant Motor Scale em lactentes nascidos prematuros. J Pediatr. 2008;84(5):442-8. doi: 10.2223/JPED.1829

18. Silva LP, Maia PC, Lopes MMCO, Cardoso MVLML. Intraclass reliability of the Alberta Infant Motor Scale in the Brazilian version. Rev da Esc Enferm. 2013;47(5):1046-51. doi: 10.1590/ S0080-623420130000500006

19. Bunce C. Correlation, Agreement, and Bland-Altman Analysis: Statistical Analysis of Method Comparison Studies. Am J Ophthalmol 2009;148(1):4-6. doi: 10.1016/j.ajo.2008.09.032

20. Giavarina D. Understanding Bland Altman Analysis. Biochem Medica. 2015;25(2):141-51. doi: 10.11613/BM.2015.015 